This is the accepted version of the following article: C. Y. Ji and Y. Y. Yan, 'A molecular dynamics simulation of liquid-vapour-solid system near triple-phase contact line of flow boiling in a microchannel', Applied Thermal Engineering, Vol 28(2-3): 195-202, first published online April 10, 2007.

The version of record is available online via doi:

http://dx.doi.org/10.1016/i.applthermaleng.2007.03.029

Published by Elsevier. Copyright (C) 2007 Elsevier Ltd. All rights reserved. 


\title{
A molecular dynamics simulation of liquid-vapour-solid system near triple- phase contact line of flow boiling in a microchannel
}

\author{
C.Y. Ji and Y.Y. Yan* \\ School of the Built Environment, University of Nottingham, Nottingham NG7 2RD, UK
}

\begin{abstract}
In this paper, the liquid-vapour-solid system near triple-phase contact line in a microchannel heat sink is studied numerically. Molecular dynamics (MD) method is employed aiming to get a microscopic insight into the complex liquid-vapour-solid system. In the present model, the Lennard-Jones potential is applied to mono-atomic molecules of argon as liquid and vapour, and platinum as solid substrates, to perform a simulation of non-equilibrium molecular dynamics. The results of numerical simulation suggest that for a complete wetting system, such as argon on a platinum substrate, there is a non-evaporating liquid film with thickness in nanometres existing on the heating solid surface. The minimum film thickness near the triplephase contact line under different conditions of the substrate temperature is predicted. The thickness of such an ultra-thin liquid film varies only slightly with the number of argon molecules but decreases with the increase of heating substrate temperature. The decrease in potential energy toward the region near the heating wall is considerably large indicating that the interactions of solid and liquid molecules are very strong.
\end{abstract}

Keywords: microchannel; molecular dynamics simulation; ultra-thin liquid film; flow boiling

\footnotetext{
Corresponding author. Tel.: +44 115951 3168; fax: +44 115951 3159. Email: yuying.yan@ nottingham.ac.uk
} 


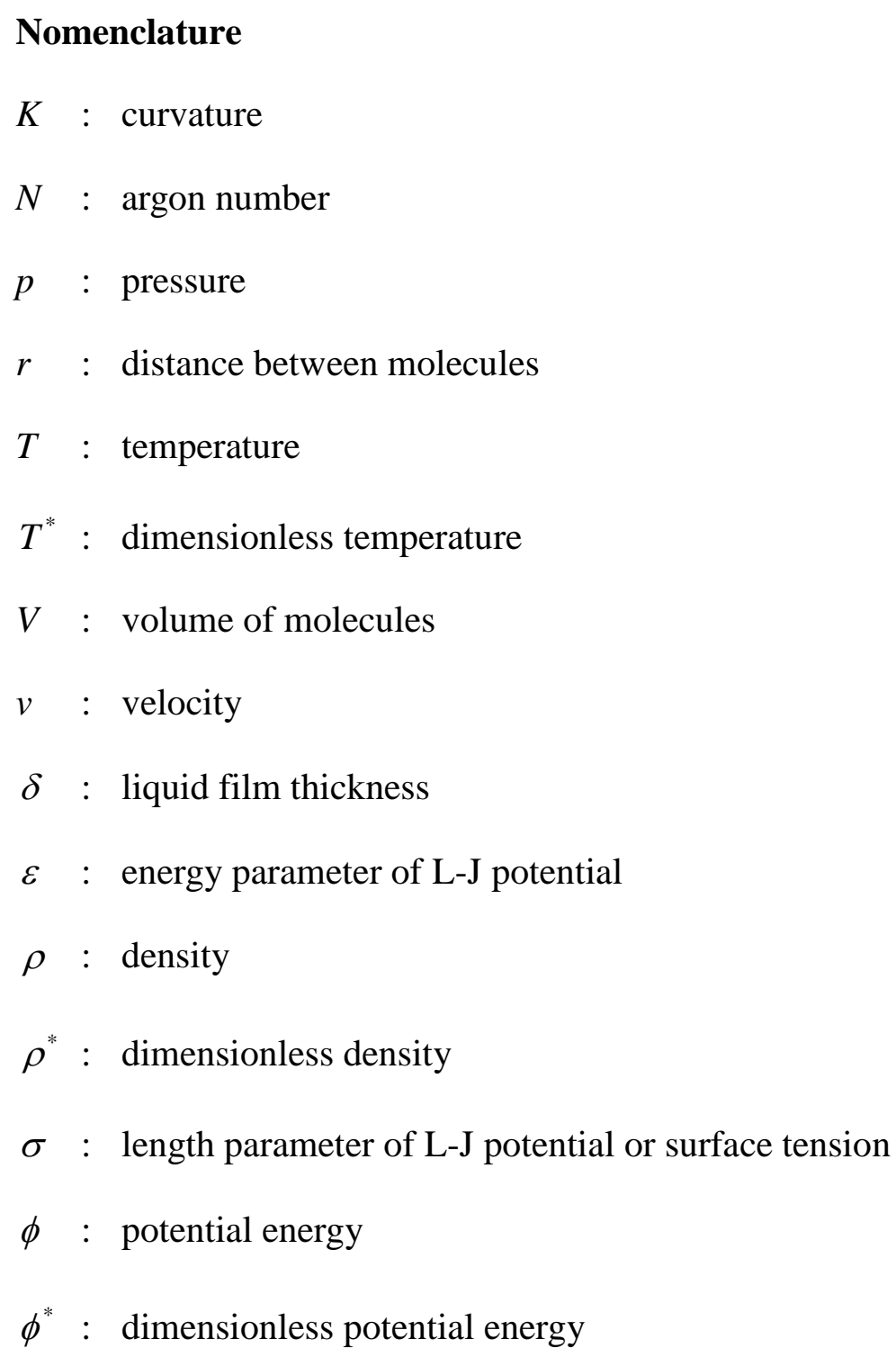




\section{Introduction}

With recent development of the modern electronic and computer industry, the miniaturisation of chips and the increase of processing speed have resulted in a large reduction in heat transfer surface area, thus a very high heat flux is normally generated from a small surface area resulting in a very high temperature. Indeed, this has significantly increased the demand for the power of cooling chips. Such increased demand for intensive cooling of compact and micro electronic devices, such as the CPUs of high performance computers, has forced engineers to develop a new generation of heat sinks at micro scales.

Phase change heat transfer, such as evaporation or boiling, allows accommodation of very high heat flux at relatively low wall superheats. Therefore, it can be used as a highly effective means to remove the extra heat flux generated in an electronic system. The study of phenomena of heat and mass transfer near a triple-phase contact line in evaporation or boiling has attracted a great deal of attention from many researchers in recent decades [1-7]. Such phenomena often occur in a number of circumstances such as capillary flow in grooves of heat pipes or evaporators, flow in inclined partially submerged plates, movement of liquid layers underneath vapour bubbles and columns. In fact, microchannel heat sinks with phase change heat transfer have increasingly become commonly-used highly efficient devices for electronic cooling [8].

In recent years, the studies on computational modelling of flow and heat transfer of flow boiling in a microchannel have become very active in academic societies. From the macroscopic standpoint, all variables and parameters are treated as continuous while being mathematically calculated in meshes no matter how small in length scale. The region around the contact line can usually be divided into micro-layer region and macro-layer region. In the previous study, these 
two regions have to be analyzed or computed separately $[2,3,5]$. The heat transfer in micro-layer region is normally analyzed theoretically, and the heat transfer in macro-layer is investigated using various numerical or theoretical analysis methods. It is impossible to solve the heat transfer problem in the micro-layer by conventional computational fluid dynamics (CFD) method because the length scale of the layer is too small. In addition, when the characteristic length of a fluid domain is smaller than an intermolecular distance, the macroscopic Lifshitz theory is ineffective and the continuum media assumption may break down $[9,10]$. The length scale in the vicinity of triple-phase contact line is so small that a microscopic calculation is needed to provide new information to complement the macroscopic approach.

As for the microscopic calculation approach, molecular dynamics (MD) and Monte Carlo (MC) simulation method are often used to investigate the physical phenomena of fluid-solid interactions with very small length scale [11-18]. Using non-equilibrium molecular dynamics simulation with a temperature gradient imposed, Xue et al. have demonstrated that the layering of a simple monoatomic liquid does not have any significant effect on liquid-solid interfacial thermal resistance [11]. Freund studied a two-dimensional (in the mean) liquid drop centred on a cold spot on an atomically smooth solid wall with evaporating menisci extending from it onto hotter regions of the wall [12]. Ohara et al. simulated numerically liquid argon (without vapour phase) between two solid walls and studied the intermolecular energy transfer at the solid-liquid interface [13]. Yi et al. performed molecular dynamics simulations of vaporization phenomena of an ultra-thin layer of argon on a platinum surface. They simulated the entire vaporization process for two temperature cases and the reverse process, the condensation, after complete evaporation [14]. Effects of surface wettability on the behaviour of liquid atoms near a solid boundary were also studied by using MD simulation methods [15, 16]. Wemhoff et al. explored a hybrid approach to investigate a thin liquid argon film on a solid surface by combining a deterministic 
molecular dynamics simulation of the liquid regions with a stochastic treatment of the far-field vapour region boundary [17]. Liu [18] has employed MC method to study Lennard-Jones liquid film and its vapour with a consideration of attractive and short-ranged wall potential near the liquid-solid interface.

This paper aims to study liquid-vapour-solid system in the region near triple-phase contact line of flow boiling in a microchannel heat sink. The molecules behaviour of mono-atomic argon in a liquid-vapour-solid system is simulated numerically by the MD method. In this way, it is investigated from a physical-chemical perspective instead of fluid-dynamical perspective to understand the effect of the solid heating wall on the liquid film in a completely wetting system. In addition, the liquid-vapour-solid system in the region near triple-phase contact line is studied by changing the fluid molecule number, the heating solid wall temperature, and the simulation cell size. The overall aims of this paper are to get a microscopic insight into the complex liquidvapour-solid system and a fundamental understanding of the physical mechanism, which will be helpful for the development and potential final application of a microchannel phase change heat sink.

\section{Microchannels heat sink}

Fig. 1 shows the ultra-thin liquid film structure in the vicinity of triple-phase contact line in a microchannel heat sink, which consists of several parallel micro-channels with a rectangular cross section. The working fluid flowing in microchannels removes heat energy by evaporation and boiling. Heat from the heater surface is conducted into the macro/micro liquid layer surrounding the vapour and is utilized in evaporation at the liquid-vapour interface. The liquidvapour-solid triple-phase contacting structure for evaporation and boiling can be deduced as 
shown in Fig. 2. The force balance at the liquid-vapour interface of an evaporating ultra-thin film is expressed as

$$
\rho_{V} v_{V}^{2}-\rho_{L} v_{L}^{2}=p_{L}-p_{V}+\sigma K-p_{D}
$$

where the momentum change across the liquid-vapour interface is balanced by liquid-vapour pressure difference, surface tension force and disjoining pressure. $p_{L}$ stands for the pressure in the liquid side near the liquid-vapour interface, $p_{V}$ is the pressure in the vapour side near the interface (a few molecular mean free paths away in vapour phase) (Fig. 2). $p_{D}$ is the pressure resulting from the attractive force (van der Waals force) between the solid-liquid molecules. For the purpose of simplification, the disjoining pressure of non-polar liquids is written as: $p_{D}=-A / \delta^{3}$; where A is Hamaker constant represented by $A=\pi^{2} C n_{S} n_{L} ; \delta$ is the thickness of liquid film; $n_{S}$ and $n_{L}$ are number densities of solid and liquid; $C$ comes from purely attractive intermolecular potential $w(r)=-C / r^{6}$. In the region near contact line, the curvature is so small that the influence of surface tension can be neglected; therefore the disjoining pressure plays a dominant role in Eq. (1); the value of disjoining pressure reflects the strength of intermolecular attractive force. In the present MD modeling, the basic molecular interactions which cover not only the attractive but also the repulsive forces are considered to investigate the microscopic insight of intermolecular forces and their effects on physical phenomena in the region near the contact line. 


\section{The simulation system and method}

The well-known model fluids used in MD include the hard spheres fluid, the square well fluid and the Lennard-Jones (L-J) fluid. The hard spheres fluid does not exhibit the transition of vapour-liquid phases because only repulsive interactions are present. The square well fluid, whose potential incorporates both repulsive and attractive forces between molecules but in a very simple way, is normally applied in simple atomic systems because of its simplicity and analytic tractability. On the other hand, the L-J fluid shows the transitions between vapour-liquid, solidliquid and solid-vapour phases, and the critical and triple points. Therefore, the L-J fluid is regarded as a suitable reference fluid for modelling properties of real fluids [19].

In the present investigation, a system with L-J fluid confined between two solid walls is studied using non-equilibrium molecular dynamics (NEMD) simulation method. Fig. 3 shows a snapshot of the system at steady state. This liquid-vapour-solid system is a special case of the left-hand side of Fig. 2 with an opposing cold wall, so that a gradient of conditions develops across the gap and the fluxes of molecules entering and leaving the hot and cold films are equal. The size of the cell is of $5.83 \times 3.85 \times 7.22 \mathrm{~nm}^{3}$, including both the two solid walls and the fluid confined between them. The distance between the two walls (in $z$ direction excluding the solid walls thickness) is $5.41 \mathrm{~nm}$; other cases with enlarged cell size are also simulated in this paper. Periodic boundary conditions are applied in $x$ and $y$ directions. All the variables including density, potential energy are computed in the slices of $\mathrm{x}$-y planes along $\mathrm{z}$ direction; so a smaller length scale in y direction was used to save computing time and storage.

The current simulation predicts the minimum film thickness in the triple-phase contact line under different conditions of the substrate temperature. Here the minimum thickness is an important parameter in theoretical analysis [20]. It is important to study the liquid film near the 
contact line, as the minimum film thickness is the necessary boundary condition for the analysis of the right part of Fig. 2 (which shows a qualitative sketch of the relative size of the simulation region of the contact line). In the MD simulation, although the physics on the right-hand side and the left-hand side of the contact line is not symmetry, the ultra-thin liquid film (thickness in nanometres) does extend from the left-hand to the right-hand side of the contact line to some distance [7]. Moreover, during evaporation and boiling, contact lines are usually advancing or receding relative to the solid substrate (When liquid in microchannel evaporate into vapour, the contact line recedes in $+x$ direction in Fig.2; when the supplied liquid comes into the microchannel, the contact line advances in $-\mathrm{x}$ direction). Therefore, our MD simulation system has a wider range (x direction in Fig. 2) of applicability in the region near triple-phase contact line: from the viewpoint of film thickness, our MD system is also applicable for the right-hand side of Fig. 2 near the contact line (with cooling wall included in simulation system).

For both the fluid and the solid molecules, the L-J potential function is used to calculate the intermolecular forces. This is an appropriate first step to understand the phenomenological influence of realistic atomic granularity on the liquid-vapour-solid system and suchlike [12]. The L-J potential is known to give a quantitatively reasonable description of liquid argon [10] whose molecular is of mono-atom type. Therefore, for the sake of physical understanding, in the present study, argon is used as the L-J fluid with the following potential parameters: $m_{L}=6.63 \times 10^{-26} \mathrm{~kg}$, $\sigma_{L}=0.3405 \mathrm{~nm}, \varepsilon_{L}=1.67 \times 10^{-21} \mathrm{~J}$. The solid wall is represented by four layers of face centred cubic (fcc (111), see the inset of Fig. 3) surface of harmonic platinum molecules with parameters as: $m_{S}=3.24 \times 10^{-26} \mathrm{~kg}, \quad \sigma_{S}=0.2475 \mathrm{~nm}, \varepsilon_{S}=8.35 \times 10^{-20} \mathrm{~J}[15,21]$. This type of crystalline structure is quite stable so it is not necessary to introduce any additional phantom molecules with springs holding the solid molecules at the crystalline sites. A similar method was reported by Xue et al. [12]. 
The potential function between solid and liquid molecules is represented by Lennard-Jones function as:

$$
\phi_{S L}(r)=4 \varepsilon_{S L}\left\{\left(\sigma_{S L} / r\right)^{12}-\left(\sigma_{S L} / r\right)^{6}\right\}
$$

where the Lorentz-Berthelot mixing rules [22] as $\varepsilon_{S L}=\sqrt{\varepsilon_{S} \varepsilon_{L}}$ and $\sigma_{S L}=\left(\sigma_{S}+\sigma_{L}\right) / 2$ are applied to the completely wetting system in the present study. The first term of equation (2) represents the short-range repulsive portion of the potential; the second term is the long-range attractive portion. The cut-off radius is equal to $3.5 \sigma$.

The phase diagram for argon is plotted on the basis of a Nicolas' equation [23], as shown in Fig. 4, where the cases of MD simulation correspond to the circles (open and closed) in the diagram. The temperature of upper solid wall is set as a fixed value of $90 \mathrm{~K}$. The lower wall is the heater with different temperatures, corresponding to the closed circles in vertical direction. In the modelling, dimensionless density $\rho^{*}$ is defined as $N \sigma_{L}^{3} / V$, where $N$ is argon number in the system and $V$ is the volume that argon occupies; dimensionless temperature $T^{*}$ is in the form of $\kappa T / \varepsilon_{L}$, where $\kappa$ is Boltzmann constant. For the lower wall temperature fixed at $110 \mathrm{~K}$, the systems with argon molecules, respectively at 784, 1200 and 1440, are simulated and shown by the three open circles.

\section{Results and discussion}

There are many factors affecting the liquid-vapour-solid system near triple-phase contact line in a boiling system of microchannels. For the present completely wetting L-J fluid interacting with 
the ideally planar platinum surface, numerical simulations are conducted by changing the number of fluid molecules in the system, the heating solid wall temperature, and the simulation cell size. Concrete microscopic insights are obtained by studying the influence of these parameters.

\subsection{The case with different argon number}

As shown in Figs. 5 and 6, in all cases of the simulation, ultra-thin liquid films can be observed even when the heating temperature is close to the critical temperature point of argon, which is about $158 \mathrm{~K}$ as it can be identified from Fig. 4. Liquid molecules in the film are adhered on the solid wall as a result of strong interactions of intermolecular forces in the completely wetting system. Obviously, such liquid molecules which carry extra energies cannot overcome the barrier of long-range attractive forces in the well wetting solid-liquid system. In addition, the wall-induced local surface morphology of the liquid film appears to be an evenly distributed solid-like structure.

Fig. 5 shows the ultra-thin liquid film of the system with different number of argon molecules under the same temperature condition. For a fixed lower wall temperature at $110 \mathrm{~K}$, the systems respectively with 784, 1200 and 1440 molecules are simulated. It can be seen that, for a given superheat, the variation of ultra-thin liquid film thickness with different number of molecules is very small. With the increase of argon number, extra molecules are accumulated near the cooling wall.

\subsection{The case with different heating wall temperature}

Fig. 6 shows the liquid film of a system of 1200 molecules under different temperature conditions. When the heating temperature reaches to $210 \mathrm{~K}$, the liquid film still exists but its thickness is decreased to the order of only one layer of argon molecules. 
From the density distribution of argon molecules, it can be seen that the densities in $z$ direction exhibit an oscillation structure (Fig. 7). Different regions of the liquid, interface and vapour are shown in Fig. 7. The liquid film thickness is defined as the distance from the wall to the centre of liquid-vapour interfacial region and can be calculated from the density distributions. The minimum film thickness of the triple-phase contact line under different conditions of the substrate temperature is predicted by the current simulation. To determine the effects of solid wall superheat on film thickness, the thickness of the thin film formed on the heated completely wetting surface are summarized in Fig. 8. Fig. 8 shows the film thickness variation with different temperatures. The discrete dots are thickness specified from MD results; the curve is a fitted line from these thickness data. It can be seen that the curve is fitted quite well except that there are two dots dropping out of the curve. This deviation may result from the uncertainty in specifying the film thickness. The liquid-vapour interface region is specified through density distribution such as Fig. 7, which will lead to some uncertainty. More precise methods will be developed to specify the film thickness in our work of next step. It is noted that the liquid film thickness is within 2 nanometres and has a decreasing tendency with the increase of heating temperature. This means that more liquid molecules may escape from the attractions of solid as the heater wall temperature continue to rise.

The solid heating wall effect can also be seen from potential energy distributions in the nearwall liquid film of the completely wetting system as plotted in Fig. 9, where the dimensionless potential energy $\phi^{*}$ is calculated on the basis of equation (2) but normalised by $\varepsilon_{L}$, i.e. $\phi^{*}=\phi_{S L}(r) / \varepsilon_{L}$. Fig. 9 shows that the potential energy increases notably with heating temperature. Compared with the result of free film (a liquid film bounded on both sides by vapour) [24], the potential energy curve of the liquid film, bounded by vapour on one side and by solid on the other side, shows a considerably steeper decrease toward the region near the heating 
wall due to the effect of strong interactions between solid and liquid molecules. The oscillation of density in Fig. 7 shows the strong wall-induced effect on the liquid film, whereas the smooth curve of potential energy in Fig. 9 indicates the substantial smooth change of potential energy field.

Fig. 10 shows three cases of snapshot of molecular distributions of argon in the whole simulation system and within the layer in the vicinity of the heating platinum wall (side view and top view). It shows that there is more than one layer of molecules (Fig. 10a) when the heating wall is at $110 \mathrm{~K}$. When the temperature increases to $210 \mathrm{~K}$, only one layer of molecules is adhered on the wall. It is shown in Fig. 10b that these molecules exhibit an even distribution in a honeycomb-like way. In Fig. 10c, the side and top views are cut off from the same z position (not from the interface) as in Fig. 10b to make a comparison between Figs. 10b and 10c. It is surprised to note from the figure that the molecules do not totally evaporate away even when the heating temperature rises to an extremely high value such as $600 \mathrm{~K}$. Nevertheless, compared with the cases of lower heating temperature, the molecules distribute rather sparsely and dry spots can be identified from a top view shown in Fig. 10c where the platinum surface is exposed directly not covered by argon molecules. Indeed, this reflects the extraordinarily strong interaction forces between solid and fluid molecules for a completely wetting system.

\subsection{The case with different cell size}

NEMD simulations are performed with different cell size in $x$ and $z$ directions. A snapshot of molecules distribution for an enlarged cell in $z$ direction is shown in Fig. 11a, in which, the cell gap between two walls is three times larger than the previous defined $5.41 \mathrm{~nm}$, the number of argon molecules is 1200 , and the heating and cooling wall temperatures are $110 \mathrm{~K}$ and $90 \mathrm{~K}$ respectively. It is clearly shown that although there is a larger space for the evaporated argon 
molecules to escape, the liquid film thickness near the heating wall still remains almost the same level as shown in Fig. 5 (the middle figure). While, for the second case in which the cell length in $x$ direction is twice of the previous defined length and the number of argon molecules confined between the two solid walls is increased to 2400 , the snapshot of molecules distribution given in Fig. 11b shows that the liquid film thickness remains almost unchanged when the cell width is changed.

\subsection{With different solid energy parameters}

In the above MD simulation, the solid energy parameter about 50 times larger than the liquid energy parameter $\left(\varepsilon_{S}=50 \varepsilon_{L}\right)$ has been applied. Additionally, a case study for the solid energy parameter of 10 times larger than the liquid energy parameter $\left(\varepsilon_{S}=10 \varepsilon_{L}\right)$ is also carried out and the results are presented in Fig. 12. Both of the energy parameters used can secure the face centred cubic crystalline structure in stable states well below the according melting points. It can be noted from the snapshot in Fig. 12 that more argon molecules evaporate away from the solid surface but the liquid film, although becomes a bit thinner, still remains adhering on the solid. Indeed, this is consistent with the previous discussion in which even at a very high temperature, the thin liquid layer is still adhered on the heating surface.

\section{Conclusions}

Numerical simulations based on the nonequilibrium molecular dynamics (NEMD) are performed to investigate liquid-vapour-solid system near triple-phase contact line of flow boiling in a microchannel. The main findings can be summarised as follows: 
1) For a completely wetting system of mono-atomic fluid and substrates, an ultra-thin liquid film in nanometres is adhered on heating surface even when the surface temperature reaches above the critical temperature.

2) The minimum film thickness near triple-phase contact line under different conditions of the substrate temperature is predicted to be within $2 \mathrm{~nm}$.

3) For the systems with different number of argon molecules, the film thickness remains almost the same if the heater temperature is constant.

4) The liquid film thickness decreases with the increase of wall heating temperature. However, a complete evaporation does not take place when the solid wall superheat is extremely high.

5) The decrease in potential energy toward the region near the heating wall is considerably large indicating that the interactions of solid and liquid molecules are very strong.

\section{Acknowledgements}

The project is supported by the UK EPSRC under grant EP/D500125/01. The authors would like to thank Prof. T. Tsuruta and Prof. G. Nagayama at Kyushu Institute of Technology, Japan, for useful discussions on developing the MD code.

\section{References}

[1] P.S. Ayyaswamy, I. Catton, D.K. Edwards, Capillary flow in triangular grooves, ASME Journal of Applied Mechanics 41 (2) (1974) 332-336. 
[2] Q. Bai, Y. Fujita, Numerical simulation of the growth for a single bubble in nucleate boiling, Thermal Science \& Engineering 7 (4) (1999) 45-53.

[3] D. Khrustalev, A. Faghri, Fluid flow effects in evaporation from liquid-vapor meniscus, ASME Journal of Heat Transfer 118 (3) (1996) 725-730.

[4] A. Mirzamoghadam, I. Catton, A physical model of the evaporating meniscus, ASME Journal of Heat Transfer 110 (1) (1988) 201-207.

[5] G. Son, V.K. Dhir, N. Ramanujapu, Dynamics and heat transfer associated with a single bubble during partial nucleate boiling on a horizontal surface, ASME Journal of Heat Transfer 121 (3) (1999) 623-631.

[6] P. Stephan, J. Hammer, A new model for nucleate boiling heat transfer, Warme- und Stoffubertragung 30 (2) (1994) 119-125.

[7] P.C. Wayner, Intermolecular forces in phase-change heat transfer: 1998 Kern Award Review, AIChE Journal 45 (10) (1999) 2055-2068.

[8] I. Mudawar, Assessment of high-heat-flux thermal management schemes, IEEE Components \& Packaging Technology 24 (2) (2001) 122-141.

[9] D. Bhatt, J. Newman J, C.J. Radke, Molecular simulation of disjoining-pressure isotherms for free liquid, Lennard-Jones thin films, Journal of Physical Chemistry B 106 (25) (2002) 65296537.

[10] J. Koplik, J.R. Banavar, Continuum deductions from molecular hydrodynamics, Annual Review of Fluid Mechanics 27 (1995) 257-292.

[11] L. Xue, P. Keblinski, S.R. Phillpot, S.U.-S. Choi, J.A. Eastman, Effect of liquid layering at the liquid-solid interface on thermal transport, International Journal of Heat and Mass Transfer 47 (19-20) (2004) 4277-4284. 
[12] J.B. Freund, The atomic detail of an evaporating meniscus, Physics of Fluids 17 (2) (2005) 022104.

[13] T. Ohara, D. Suzuki, Intermolecular energy transfer at a solid-liquid interface, Microscale Thermophysical Engineering 4 (3) (2000) 189-196.

[14] P. Yi, D. Poulikakos, J. Walther, G. Yadigaroglu, Molecular dynamics simulation of vaporization of an ultra-thin liquid argon layer on a surface, International Journal of Heat and Mass Transfer 45 (10) (2002) 2087-2100.

[15] G. Nagayama, P. Cheng, Effects of interface wettability on microscale flow by molecular dynamics simulation, International Journal of Heat and Mass Transfer 47 (3) (2004) 501-513.

[16] J.A. Thomas, A.J.H. McGaughey, Effect of surface wettability on liquid density, structure, and diffusion near a solid surface, Journal of Chemical Physics 126 (3) (2007) 034707.

[17] A.P. Wemhoff, V.P. Carey, Molecular dynamics exploration of thin liquid films on solid surfaces. 1. Monatomic fluid films, Microscale Thermophysical Engineering 9 (4) (2005) 331349.

[18] K.S. Liu, Phase separation of Lennard-Jones systems: A film in equilibrium with vapor, Journal of Chemical Physics 60 (11) (1974) 4226-4230.

[19] M.S. Zabaloy, V.R. Vasquez, E.A. Macedo, Description of self-diffusion coefficients of gases, liquids and fluids at high pressure based on molecular simulation data, Fluid Phase Equilibria 242 (1) (2006) 43-56.

[20] J.R. Thome, Heat transfer model for evaporation in microchannels. Part I: Presentation of the model, International Journal of Heat and Mass Transfer 47 (14-16) (2004) 3375-3385.

[21] C.Y. Ji, T. Tsuruta, G. Nagayama, Effects of solid heating surfaces on nanometer-sized liquid films, Thermal Science \& Engineering 13 (2) (2005) 25-28. 
[22] M.P. Allen and D.J. Tildesley, Computer Simulation of Liquids, Clarendon Press, Oxford, 1989.

[23] J.J. Nicolas, K.E. Gubbins, W.B. Streett, D.J. Tildesley, Equation of state for the LennardJones fluid, Molecular Physics 37 (5) (1979) 1429-1454.

[24] T. Tsuruta, H. Tanaka, T. Masuoka, Condensation/evaporation coefficient and velocity distributions at liquid-vapor interface, International Journal of Heat and Mass Transfer 42 (22) (1999) 4107-4116. 


\section{List Figure Caption - C.Y. Ji and Y.Y. Yan}

Fig. 1: Evaporating region in Microchannels Heat Sink

Fig. 2: Liquid-vapour-solid contact structure in the vicinity of triple-phase contact line

Fig. 3: Snapshot of NEMD simulation system

Fig. 4: Phase diagram of argon and MD simulation cases

Fig. 5: Snapshots of different Ar molecule number systems

Fig. 6: Snapshots of 1200 Ar molecules system with different heating temperatures

Fig. 7: Density distribution of 1200 Ar molecules system (90K-110K)

Fig. 8: Film thickness at different wall temperature

Fig. 9: Potential energy distribution of the liquid film near the heating wall of different temperatures

Fig. 10: Snapshots of argon molecules distribution near above the heating wall with different heating temperatures (1200 Ar molecules in the system)

Fig. 11: Snapshot of Ar-Pt systems of enlarged cell size

Fig. 12: Snapshot of 1200 Ar molecules system with heating temperature of $210 \mathrm{~K}$ (only the heating wall and nearby fluids are shown) 


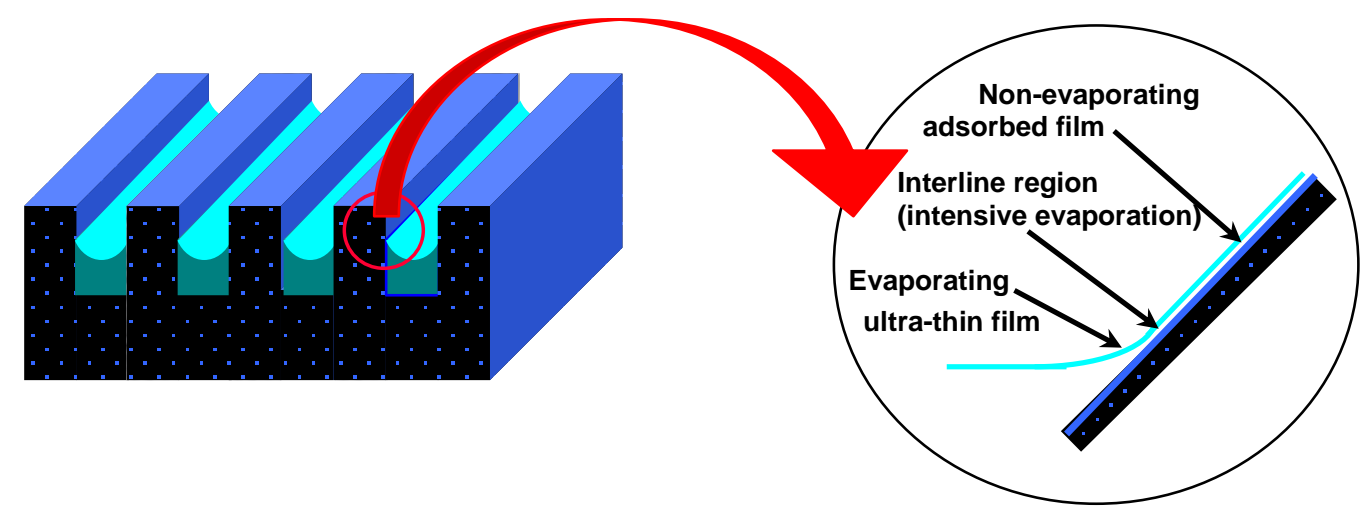

Fig. 1. Evaporating region in Microchannels Heat Sink

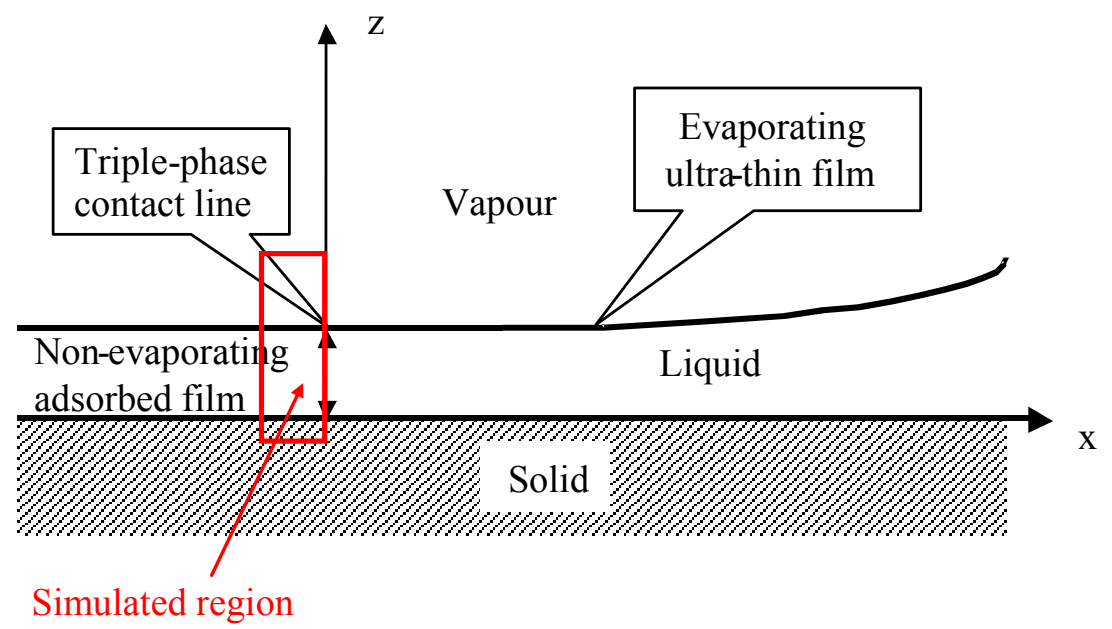

Fig. 2. Liquid-vapour-solid contact structure in the vicinity of triple-phase contact line 


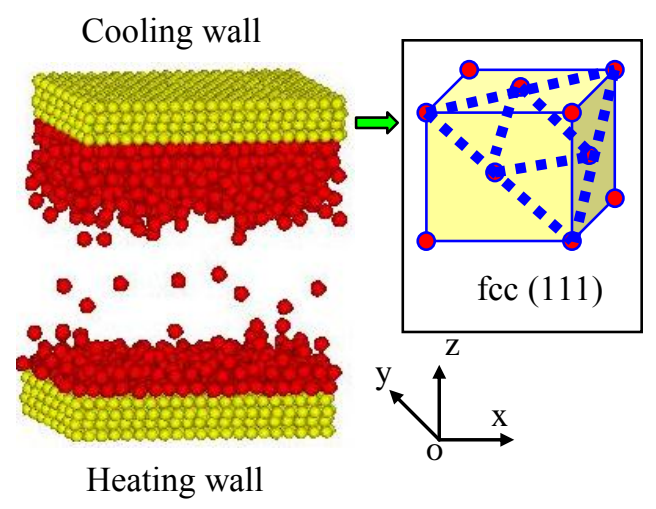

Fig. 3. Snapshot of NEMD simulation system

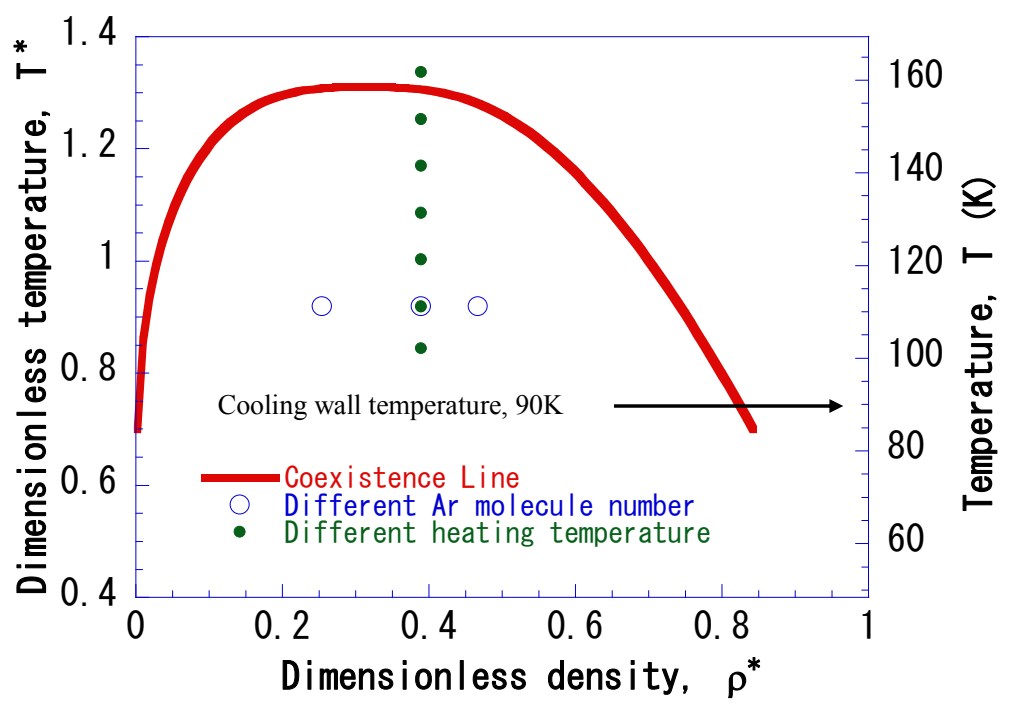

Fig. 4. Phase diagram of argon and MD simulation cases 


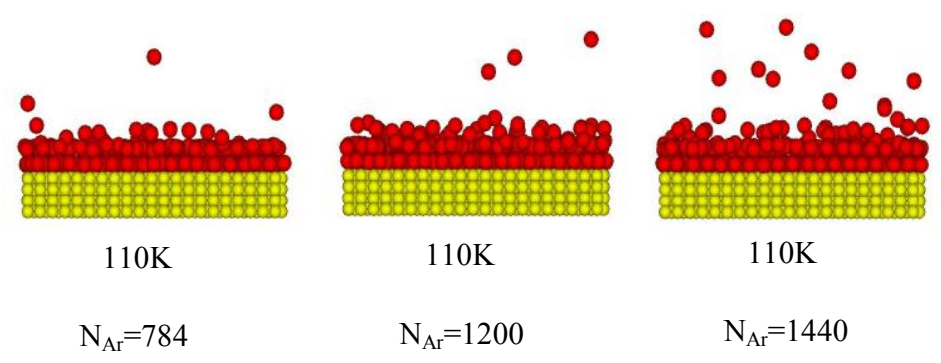

Fig. 5. Snapshots of different Ar molecule number systems (only the heating wall and nearby fluids are shown)

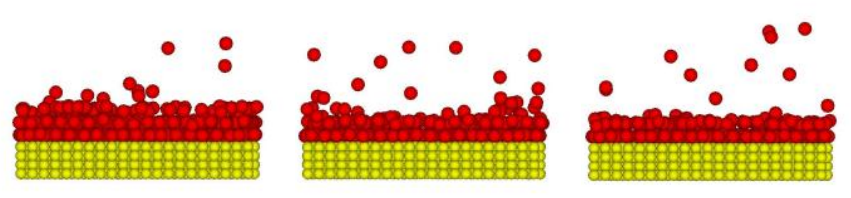

$100 \mathrm{~K}$

$120 \mathrm{~K}$

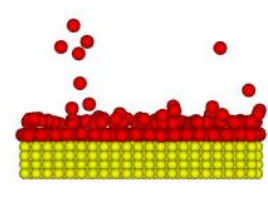

$140 \mathrm{~K}$

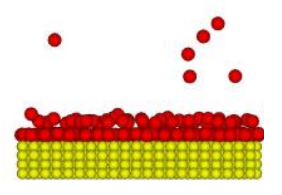

$160 \mathrm{~K}$

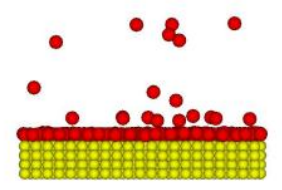

$210 \mathrm{~K}$

Fig. 6. Snapshots of 1200 Ar molecules system with different heating temperatures (only the heating wall and nearby fluids are shown) 


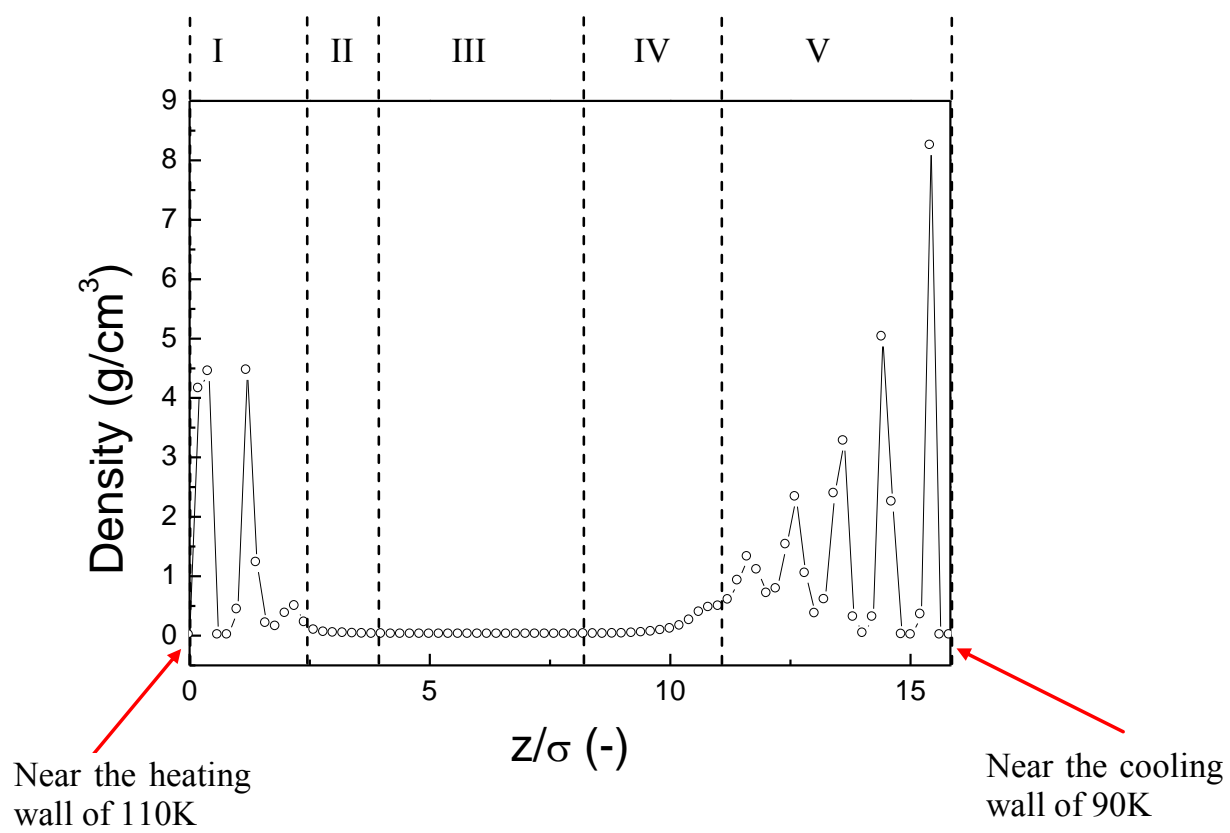

I, V: Liquid film

II, IV: Liquid-vapour interface

III: Vapour

Fig. 7. Density distribution of 1200 Ar molecules system (90K-110K) 


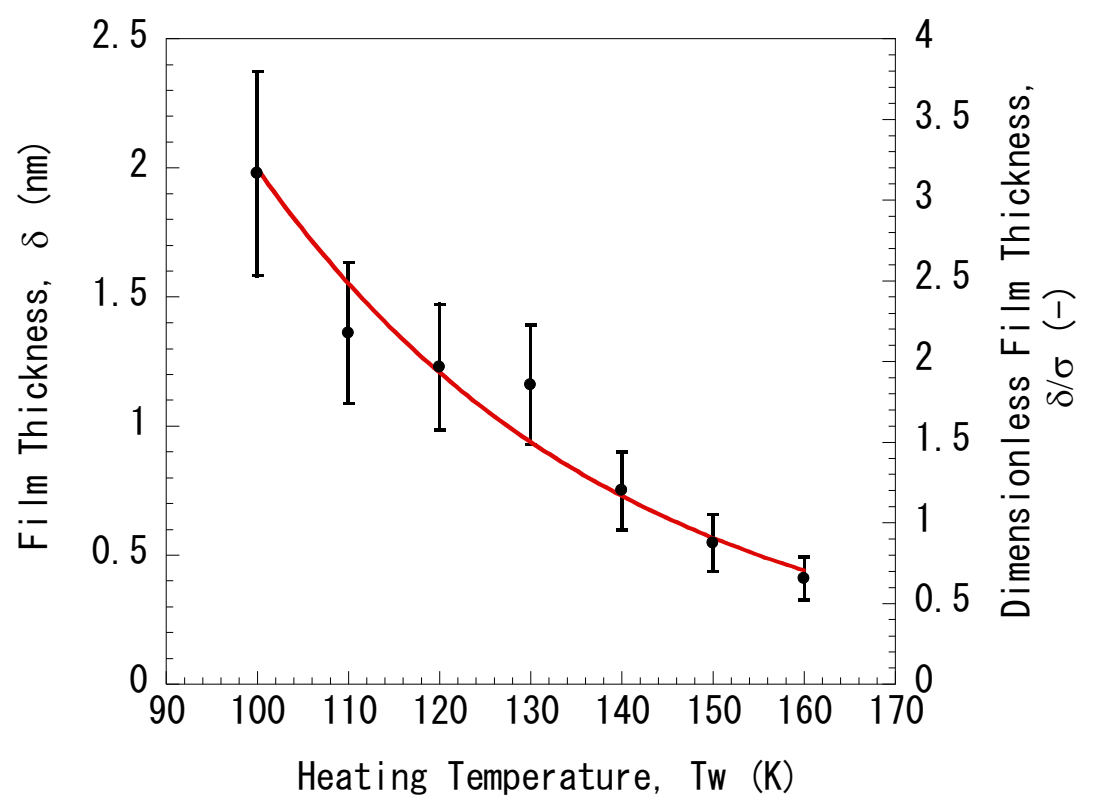

Fig. 8. Film thickness at different wall temperature (20\% error bar) 


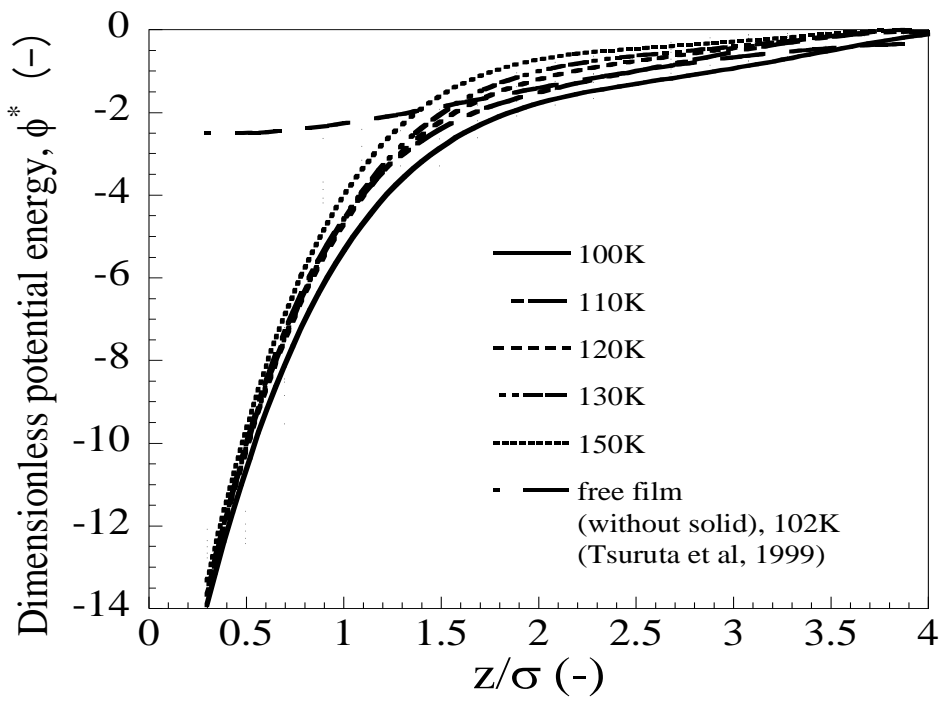

Fig. 9. Potential energy distribution of the liquid film near the heating wall of different temperatures 

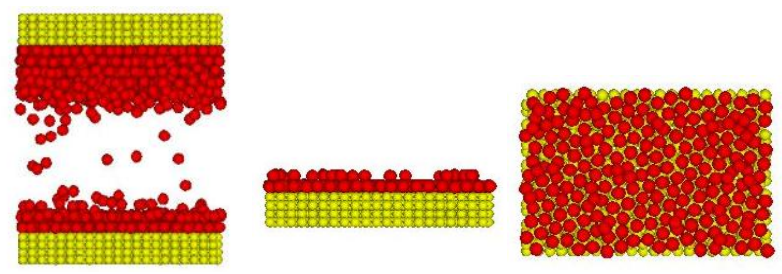

Side view Top view

(a) $\mathrm{T}_{\mathrm{W}}=110 \mathrm{~K}$

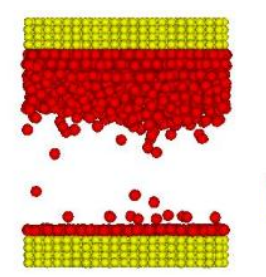

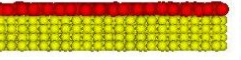

Side view

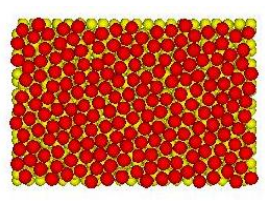

(b) $\mathrm{T}_{\mathrm{W}}=210 \mathrm{~K}$
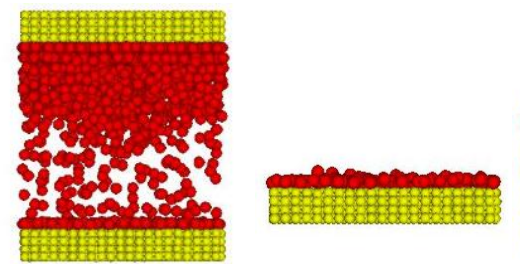

Side view

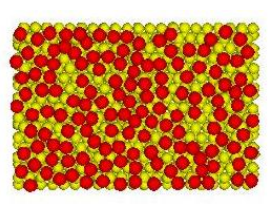

Top view

(c) $\mathrm{T}_{\mathrm{W}}=600 \mathrm{~K}$

Fig. 10. Snapshots of argon molecules distribution near above the heating wall with different heating temperatures (1200 Ar molecules in the system) 

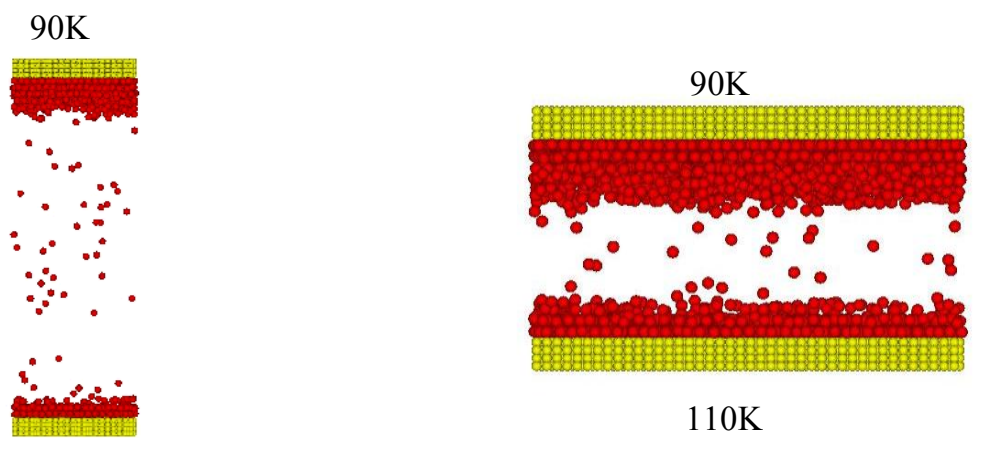

$110 \mathrm{~K}$
(a) $\mathrm{N}_{\mathrm{Ar}}=1200$
(b) $\mathrm{N}_{\mathrm{Ar}}=2400$

Fig. 11. Snapshot of Ar-Pt systems of enlarged cell size

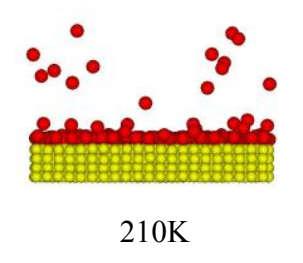

Fig. 12. Snapshot of 1200 Ar molecules system with heating temperature of $210 \mathrm{~K}$ (only the heating wall and nearby fluids are shown) 